\section{Pretreatment of Micro- projectiles to Improve the Delivery of DNA in Plant Transformation}

\section{BioTechniques 29:246-248 (August 2000)}

Accelerated metal particles coated with DNA are extensively used for introducing genes into intact plants and animals, tissues, cells and organelles (4). Of the various methods used to accelerate microparticles, gold microprojectiles driven by the discharge of compressed helium are employed most commonly. A principal advantage of using gold particles, instead of others like tungsten, is their uniform size, regular shape and chemically inert nature. A major difficulty encountered in using microprojectiles, especially gold particles, is that the particles agglomerate, which leads to their nonuniform spread following bombardment on the target tissue.

The tendency for adhesion results in clumps sticking to the walls of poly-

Table 1. Effect of the Modified Method of Preparation of Gold Particles on Transient Expression of gusA in Tobacco Leaves

\begin{tabular}{|c|c|c|c|c|}
\hline \multirow[b]{2}{*}{ Treatment } & \multicolumn{2}{|c|}{ Old particles } & \multicolumn{2}{|c|}{ New particles } \\
\hline & Ethanol & Isopropanol & Ethanol & Isopropanol \\
\hline \multicolumn{5}{|c|}{ A. Gus activity (pmol MU formed/h/mg protein) } \\
\hline Heated & $633 \pm 107$ & $758 \pm 33$ & $689 \pm 97$ & $1395 \pm 93$ \\
\hline Non-Heated & $18 \pm 11$ & $167 \pm 67$ & $30 \pm 13$ & $326 \pm 28$ \\
\hline \multicolumn{5}{|c|}{ B. Gus activity (number of blue spots/shot) } \\
\hline Heated & $5312 \pm 764$ & $7117 \pm 119$ & $5986 \pm 786$ & $8976 \pm 697$ \\
\hline Non-Heated & $112 \pm 59$ & $1365 \pm 238$ & $178 \pm 102$ & $2618 \pm 262$ \\
\hline
\end{tabular}

Each data point gives mean \pm SD of six independent bombardment events.

propylene tubes. This leads to variable adsorption of DNA on the particles, uncontrollable shot-to-shot variations and irreparable damage to target tissue $(3,5)$. The use of certain brands of microcentrifuge tubes has been suggested (4) to reduce the particles sticking to surface of the tube. Repeated vortex mixing, ultrasonication and the use of glycerol or polyethylene glycol reduce aggregation marginally. To make corrections for variation among results from individual bombardment events, the readings are normalized by cobombardment with a reporter gene that acts as an internal standard (1). However, uniform delivery of the microprojectiles for reproducible results continues to be a major problem (4).

We describe two simple modifications to particle and DNA preparation protocols that result in substantial enhancement and reproducibility of biologically functional delivery of DNA using gold particles. In the experiments described here, the improved protocol was compared to transient expression of the gusA reporter gene (cloned in a 6 $\mathrm{kb}$ plasmid), following its bombardment on tobacco leaves. The simple modifications in the protocol are comprised of heating the gold particles before coating with DNA and replacing ethanol with isopropanol during the steps for coating.

In a standard procedure developed by Sanford et al. (4) and recommended by Bio-Rad Laboratories (Hercules, CA, USA), $3 \mathrm{mg}$ gold particles are placed in a microcentrifuge tube and vortex mixed for $3 \mathrm{~min}$ in $0.5 \mathrm{~mL} 70 \%$ ethanol (v/v). The suspension is held at room temperature for $15 \mathrm{~min}$, cen- trifuged (15000 rpm) for $5 \mathrm{~s}$, decanted and washed three times with $500 \mu \mathrm{L}$ sterile distilled water before suspending in $50 \mu \mathrm{L}$ sterile $50 \%$ glycerol. The suspended particles are then coated with DNA by adding $5 \mu \mathrm{L}$ DNA $(1 \mu \mathrm{g} / \mu \mathrm{L}$ in water), $50 \mu \mathrm{L}(2.5 \mathrm{M}) \mathrm{CaCl}_{2}$ and $20 \mu \mathrm{L}$ fresh spermidine $(0.1 \mathrm{M})$ in that order. The mixture is then vortex mixed for 3 $\mathrm{min}$, held at room temperature for $5 \mathrm{~min}$ and the coated particles are pelleted by pulse centrifugation. The preparation is then washed sequentially with $70 \%$ ethanol and absolute ethanol before it is suspended in $48 \mu \mathrm{L}$ ethanol. The DNAcoated particles are then vortex mixed for $1-2 \mathrm{~s}$ before placing $8-\mu \mathrm{L}$ aliquots on macrocarriers for bombardment.

In the improved procedure, all other steps were followed as described except that the gold particles were heated in a dry oven at $180^{\circ} \mathrm{C}$ overnight in a glass tube before use in the above protocol. The second modification was that ethanol was substituted with isopropanol (HPLC grade; Spectrochem, Bombay, India) in all the steps described above. The bombardment was carried out at 1100 psi on tobacco leaves, placed at $12.3-\mathrm{cm}$ target distance and using the PDS-1000He helium-driven particle gun (Bio-Rad Laboratories). The bombarded leaves were incubated on MS agar medium. The glucuronidase activity was examined histochemically by counting the number of blue spots per shot; they were also measured by quantitative fluorimetry two days after bombardment, as in published procedures (2).

For standardization of the improved protocol, the particles were examined microscopically for agglomeration at 
various stages and inspected for visual adherence to the walls of microcentrifuge tubes. On the basis of these observations, heating of gold particles was selected as a useful treatment. Other alterations, including vortex mixing and ultrasonication with or without washing with chloroform, acetonitrile, ethanol, $50 \%$ glycerol and $2 \mathrm{M} \mathrm{NaCl}$, did not give significantly improved results. Acetone reduced clumping but did not prevent the gold particles from sticking to the walls of polypropylene tubes. The use of isopropanol during preparation of microcarriers and coating of DNA gave a distinct advantage. Hence, preheating of gold particles and washing with isopropanol rather than ethanol were used to determine glucuronidase expression, using two different batches of $1-\mu \mathrm{m}$ gold particles. The old batch was received from BioRad Laboratories in July 1996 while the new batch was received in August 1999. Both batches were stored (without desiccant) at room temperature in the vials supplied by the manufacturer.
The results given in Table 1A illustrate that preheating of the particles enhanced transient expression of gusA 23and 36-fold, respectively, in the case of the new and the old batch of the particles. Using isopropanol instead of ethanol on unheated particles also improved expression by about tenfold. However, when the particles were heated in advance, washing with isopropanol instead of ethanol gave a maximum of only twofold advantage. We recommend both preheating the particles and using isopropanol to obtain the best results. Use of both modifications gave 46- and 43-fold higher GUS activity, respectively, with the new and old particles.

Histochemical visualization of the number of blue spots per shot (Table 1B) showed that the improved protocol enhanced the total number of spots by 50- and 63-fold, respectively, for the new and old particles. The increased number of spots commensurate with the increase in GUS activity substantiates that our protocol facilitates uniform spread of the particles. It does not suggest an increase in gene expression as such, but only an increase in the events of expression-proficient delivery of DNA. The particles prepared by our protocol did not stick to polypropylene tubes of a variety of local brands.

In addition to the several-fold increase in transient expression, the improved protocol provided more highly reproducible shot-to-shot results. The coefficient of variation was only $6.7 \%$ for the heated isopropanol-prepared new particles compared to $43 \%$ for the particles prepared by the conventional method. Raising the temperature of preheating gave incremental increases in expression, which reached optimum values between $150^{\circ}$ and $200^{\circ} \mathrm{C}$. For optimum results, the particles needed to be preheated for more than $9 \mathrm{~h}$. Heating by autoclaving resulted in nearly nontransformable gold particles, suggesting that heating in dry air was required to prevent agglomeration. The improved results may result primarily from the removal of closely adsorbed water molecules that may be responsible for clumping of the gold particles. Storage of preheated particles in a desiccator showed no deterioration in their transformability during the 15 days of the study.
The improvement varied from nearly 5- to 50-fold in several batches of particles obtained from nearby laboratories. The advantage of heating was less (fiveto tenfold) if the particles were imported in dry weather and kept in the desiccator immediately after arrival. We suggest that a routine step of preheating the particles, combined with isopropanol, can ensure high efficiency and reproducibility, irrespective of past handling of the particles. This improved protocol can be used in studies on gene expression. The improvement may have broader value if the increase in biologically proficient DNA delivery also leads to more stable transformation.

\section{REFERENCES}

1.Bruce, W.B., A.H. Christensen, T. Klein, M. Fromm and P.H. Quail. 1989. Photoregulation of a phytochrome gene promoter from oat transferred into rice by particle bombardment. Proc. Natl. Acad. Sci. USA 86:9692-9696.

2.Jefferson, R.A. and K.J. Wilson. 1991. The GUS gene fusion system, p. 1-33. In S. Gelvin and R. Shilperoort (Eds.), Plant Molecular Biology Manual. Kluwer Academic Publishers, Dordrecht, Germany.

3.Ratnayaka, I.J.S. and J.H. Oard. 1995. A rapid method to monitor DNA precipitation onto microcarriers before particle bombardment. Plant Cell Reports 14:794-798.

4.Sanford, J.C., F.C. Smith and J.A. Russell. 1993. Optimising the biolistic process for different biological applications. Methods Enzymol. 217:483-510.

5.Vain, P., N. Keen, J. Murillo, C. Rathus, C. Nemes and J.J. Finer. 1993. Development of the particle inflow gun. Plant Cell, Tissue and Organ Culture 33:237-246.

This work was supported by a grant from The Council of Scientific and Industrial Research and The Department of Biotechnology, Government of India. Address correspondence to Dr. Rakesh Tuli, National Botanical Research Institute, Molecular Biology \& Genetic Engineering Department, Rana Pratap Marg, Lucknow 226001, India.e-mail: rakeshtuli@hotmail.com

Received 1 November 1999; accepted 6 April 2000.

Samir V. Sawant,
Pradhyumna Kumar Singh
and Rakesh Tuli
National Botanical Research
Institute
Lucknow, India

